\title{
What Do the People Who Use the Research Results Think? Attempting Integrated Knowledge Translation in the 'Knowledge Creation' Phase
}

\author{
Andrea Rishworth ${ }^{1}$, Susan Elliott ${ }^{1}$, Jenna Dixon $^{1}$, Ann Clarke ${ }^{2}$ \\ ${ }^{1}$ Department of Geography and Environmental Management, University of Waterloo, \\ 200 University Ave W., Waterloo, ON, N2L 3G1, Canada \\ ${ }^{2}$ Department of Medicine, University of Calgary, 2500 University Dr. NW, Calgary, Alberta, T2N 1N4, Canada
}

Copyright $\mathrm{O} 2016$ by authors, all rights reserved. Authors agree that this article remains permanently open access under the terms of the Creative Commons Attribution License 4.0 International License

\begin{abstract}
Collaboration among researchers and knowledge users, referred to as integrated knowledge translation (iKT), (potentially) enhances the relevance and use of research, leading to improved policies and practice. However, iKT is often applied in idiosyncratic ways, limiting integration of both knowledge creation and action. Further, iKT is rarely used at the stage of knowledge creation. Rather, it is typically used at the stage of knowledge synthesis or dissemination. This paper reports results of one part of a larger research program undertaking iKT through the integration of knowledge users and scientists in the process of knowledge creation around food allergies in Canada. A focus group discussion with 14 knowledge users involved in an ongoing biomedical research project was conducted with the purpose to: (1) share results of recent national survey data describing the prevalence and determinants of food allergy in Canada; and (2) elicit input into the next wave of survey activity in order to ensure the relevance of the next round of data collection. Stakeholders reported a lack of relevant, credible data sources to establish accurate understandings of food allergy for constituents. Confusion and doubt around information resources underline issues of insufficient knowledge translation, hindering knowledge user's credibility as providers of important public health messages. Integrating knowledge users in the process of knowledge creation is essential to facilitate stronger communication between researchers and knowledge users, help modify approaches, and improve population health outcomes; in short, iKT makes science useful.
\end{abstract}

Keywords Integrated Knowledge Translation, Anaphylaxis, Public Health

\section{Introduction}

The co-production of knowledge, involving meaningful interaction between knowledge producers and knowledge users, is a documented influential means of enabling evidence-informed policy and practice [1-3]. With growing concern that research findings are often unsuccessfully applied in practice [4], knowledge translation (KT) and its guiding Knowledge to Action Framework ('the KTA Framework') [4] are promulgated as ways to deliver improved health care interventions that will, in turn, positively affect the health of populations.

Knowledge translation (KT) is an iterative process for improving health care practice and associated outcomes by promoting the use of research results in decision making. The definition of KT derived by the Canadian Institutes of Health Research (CIHR), and subsequently adopted by the World Health Organization, reads:

[A] dynamic and iterative process that includes synthesis, dissemination, exchange and ethically-sound application of knowledge to improve the health of Canadians, provide more effective health services and products and strengthen the health care system. This process takes place within a complex system of interactions between researchers and knowledge users which may vary in intensity, complexity and level of engagement depending on the nature of the research and the findings as well as the needs of the particular knowledge user. [5]

This definition encapsulates the 'knowledge to action' cycle that involves synthesizing knowledge, interacting with target users to assess needs and identify barriers, using that information to tailor knowledge products and select implementation strategies, and ongoing monitoring to evaluate impact [4]. This action cycle is now more commonly referred to as integrated knowledge translation (iKT) given the primary aim of facilitating continuous interaction among researchers and knowledge users. iKT involves the development of a relationship between researchers and decision-makers (i.e. clinicians, managers, policy-makers, etc.) for the purpose of engaging in a mutually beneficial project or program of research [6]. iKT is distinguished from KT through its emphasis on partnership and collaboration [4]. 


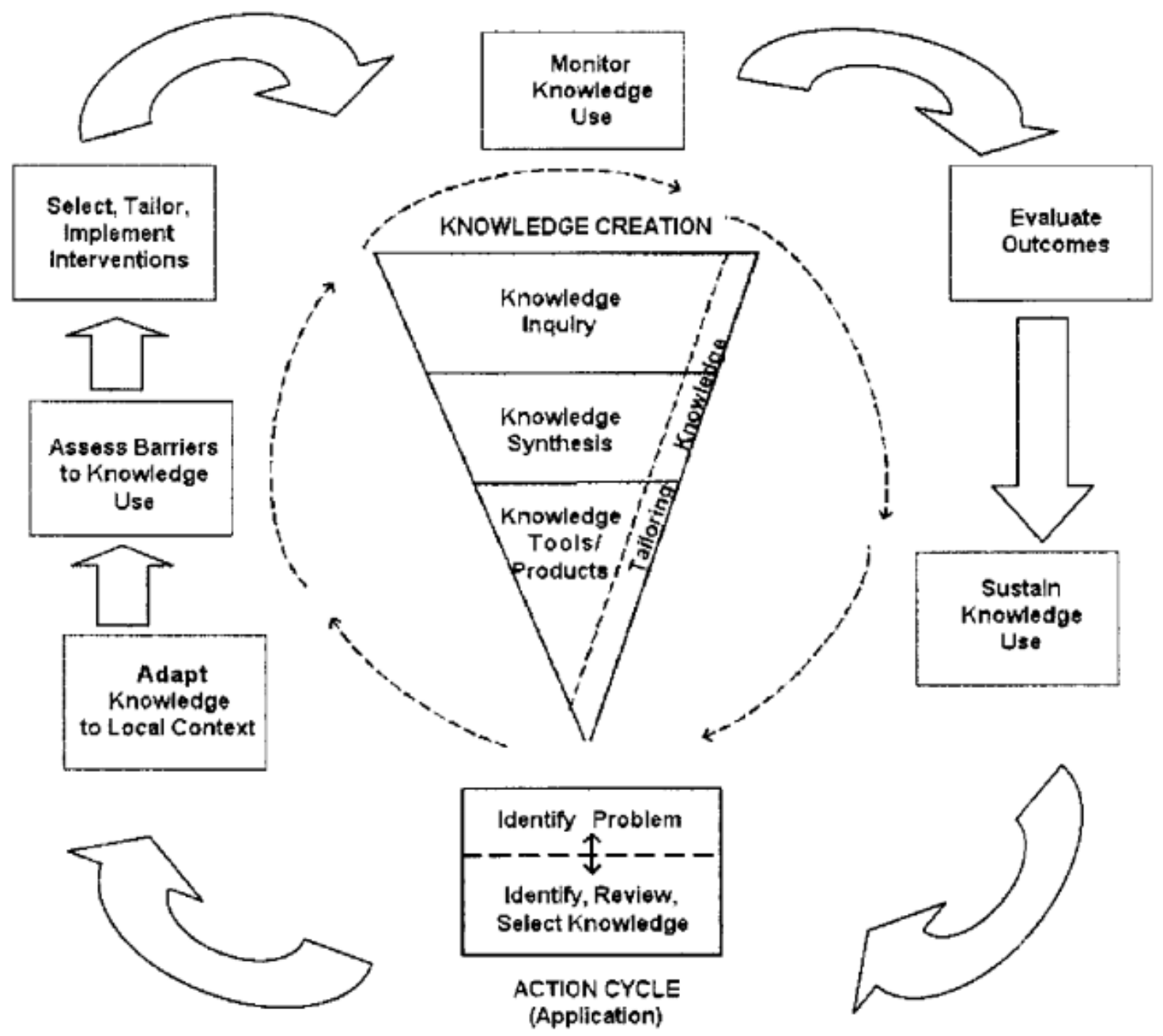

Figure 1. Knowledge to action process [4]

The use of the KTA framework (Figure 1) ascribes equivalent value to Knowledge Creation (knowledge inquiry, synthesis and the creation of products or tools) and the Action Cycle (activities leading to the implementation or application of knowledge) [4]. Collaboration, like that promoted through iKT, involves ongoing, dynamic interaction among researchers and knowledge users, representing an ideal means by which to address complex problems [7]. Within this integrated process, both phases remain permeable, although, a recent systematic review finds few examples in the literature of a comprehensive application of the framework [8].

Empirical examples of the successful use of iKT can, however, be found in the literature $[9,10]$. These range from improved communication, enhanced skills and confidence [10], and increased professional development [11]. Others indicate $\mathrm{iKT}$ enriches communication between agencies, provides the ability to identify organizational competencies and knowledge deficits, while also assisting the development and implementation of tailored training programs [12]. Despite evidence pertaining to the benefits of iKT vis-a-vis the use of research and improved health care planning, delivery and outcomes, gaps in our understanding remain; how do we - exactly - do iKT $[6,13]$.

In particular, uncertainties surround the most effective strategy to develop and sustain researcher-knowledge user partnerships [14]. A recent systematic review of the KTA Framework indicated its application in a range of idiosyncratic forms with far greater emphasis on the Action Cycle as opposed to Knowledge Creation [8] thus thwarting any comprehensive understanding of the dynamic interaction between them [13]. Yet, we do know that decision makers use research results when there is a greater linkage and exchange between the worlds of research and policy, addressing the kinds of questions of interest to policy makers [15]. Nonetheless, despite increased demand for evidence based methods and practice, this research-policy gap still remains [16]

As such, we sought the opportunity to engage knowledge users in the context of food allergies, a serious and growing public health problem [17]. In so doing, we aimed to explore the knowledge users' perceptions and experiences acquiring food allergy information in Canada. Further, we sought to attain their recommendations for future food allergy research in Canada. The questions posed during the focus group were informed by the research objectives designed to investigate i) 
experiences and perspectives acquiring information resources related to food allergy, ii) descriptions and experiences of food allergies in Canada, iii) reactions to results from the first two national surveys, and iv) recommendations for the next national survey. A knowledge user was classified as an individual who was likely to use research results to make informed decisions about health policies, programs, and/or practices (i.e. patient support and advocacy groups, private sector organizations, policy makers) [5]. Accordingly, analyzing this phase in the iKT process provides a better understanding of the ways in which knowledge users attain, experience, and perceive food allergy information, and how this informs the services they are able to provide.

\section{An iKT approach to understanding food allergies in Canada}

$7.5 \%$ of Canadians self-report at least one food allergy [18], though the prevalence differs across socioeconomic groups and geographic regions [17]. Moreover, food allergies, directly or indirectly, affect $50 \%$ of Canadian households [19]. Anaphylactic food allergies commonly occur within minutes of exposure to an allergen and can be fatal if treatment is delayed [20]. Until recently, the only treatments were strict avoidance of the allergen and immediate use of epinephrine. Recent developments in the area of oral immunotherapy to desensitize individuals so they can consume the food to which they were once allergic holds some promise for future treatment [21]. However, living with a food allergy has been shown to detrimentally affect an individual's quality of life, with many constantly negotiating physical safety and social wellbeing [22-24]. Given this context, there is pressing need for more research on the origins and treatment of food allergies, and to have that knowledge effectively mobilized by the users of that knowledge.

As part of a food allergy research program in Canada, we are working with a steering committee of knowledge users using an iKT protocol related to knowledge creation around the determinants, prevalence and experience of food allergies. The steering committee consists of representatives of patient support groups, advocacy groups and policy makers. To date, the steering committee has been working together for almost three years, with the first task having been the development of a comprehensive Terms of Reference for working together. The steering committee plays an essential role in the overall project and is constantly engaged in all phases of the research, from knowledge creation through synthesis and dissemination, with a strong commitment to the co-production of knowledge.

Members of this research team (Elliott \& Clarke) provided the first view of the landscape of food allergy in Canada. However, there is limited knowledge of the temporal trends in food allergy prevalence. Studies conducted in the US [25] and elsewhere suggest that the prevalence of food allergy, particularly in developing countries, may be increasing, but research in the UK [26] indicates that it may be stabilizing.
Accordingly, the same members of the research team (Elliott \& Clarke) will conduct another national survey in order to portray the food allergy landscape as it unfolds over time in Canada. Given the importance of integrating knowledge users in the research process, the research team conducted a focus group discussion with all members of the steering committee to enquire about their perspectives on the current data around prevalence, determinants, and experience of food allergies, and to obtain their input on what might need to be changed in the next round of data collection. These activities are based on the foundation in the literature that involving knowledge users as partners in the research process is a strong predictor that research findings will be used and that the research endeavor will achieve a greater impact [3].

\section{Methods}

While this project is part of a larger investigation looking at iKT in biomedical research, in this paper we focus on the integration of knowledge users in the process of knowledge creation, particularly centering on the knowledge users' perspectives, concerns, and needs related to food allergy information as the research team moves forward with the next stage of research. Perspectives were captured in a focus group discussion, a method often used for assessing health education messages and/or to examine individuals' experiences with health services as it allows individuals to discuss ideas openly, exchange anecdotes and comment on each other's experiences and viewpoints [27]. One week prior to the focus group, all participants received a copy of the prevalence paper that had been published in the peer reviewed literature [17]. In addition, the focus group began with a presentation of key findings by one of the authors (Elliott).

In total, $\mathrm{n}=14$ knowledge users participated, representing patient support and advocacy groups, as well as policy makers (local, regional, national). The focus group met for approximately two hours and the discussion was digitally audio-recorded with permission. The questions posed during the focus group were informed by the research objectives aimed to investigate i) experiences and perspectives acquiring information resources related to food allergy, ii) descriptions and experiences of food allergies in Canada, iii) reactions to results from the first two national surveys, and iv) recommendations for the next national survey. A checklist of topics (semi structured and open-ended questions) guided the focus group discussions and probed participants about several different topics related to the study objectives. The checklist was designed to be flexible; new questions were added as necessary during the data collection process. Examples of the questions that were asked include: "How do you access food allergy information? What are the challenges you encounter accessing information?" and "How would you explain the impact this has on your ability to provide services?" 
Audio files were transcribed verbatim for subsequent thematic analysis [28]. A thematic analysis of the data was guided by our research objectives on informants' perceptions on food allergy in Canada. Following Strauss and Corbin [29], we conducted line-by-line coding to produce textual elements which were organized into themes and sub-themes. First, a deductive approach was used to explore the data for themes elicited by way of the interview schedule. Second, themes that emerged inductively from the interview transcripts were tagged. In the end, four main themes were identified from the deductive analysis. Among and within these, several sub-themes emerged inductively from the qualitative analysis. The themes and codes were then reviewed by the first two authors for consistency, which facilitated a degree of inter coder reliability, promoting investigator triangulation, and strengthening the credibility of results [30].

\section{Results}

Results are organized around four key themes: experiences and perspectives of participants acquiring information resources, understanding and description of food allergy in Canada, reactions to the published findings, and recommendations for the next national survey. Key findings are punctuated by quotations from participants.

\section{Experiences and Perceptions of Acquiring Anaphylaxis Information Resources}

All participants reported that food allergies are very common in Canada, yet they recognized their complicated etiology and noted many challenging experiences acquiring coveted food allergy information. Participants used a variety of sources to obtain desired information ranging from common internet searches (i.e. Google searches), friends and family, to the use of medically evaluated information provided by advocacy groups (i.e. Anaphylaxis Canada, now Food Allergy Canada) and policy makers (i.e. Health Canada). One participant commented on the experience of searching for information:

Hum... I have tried to use Google and not been very satisfied with that and I find talking to other people is helpful but sometimes that's hit and miss too...it depends on who the person is. (Participant 4)

Participants revealed that depending on the type of information they required, they would pursue a variety of data sources. Additionally, they noted the capacity of their organization (i.e. size, outreach, community engagement, connectivity to researchers, public health providers, policy makers) further shaped their ability to acquire relevant, accurate information:

In terms of information, there's a huge range. I know even from the community setting, parents go to their physician, or allergist, dietitian, or the pharmacist which is becoming a more community based resource. So it depends on your outreach, where you are and what you're looking for, there are a number of different groups people can go to. (Participant 2)

Although participants commonly noted acquiring information from an array of sources (i.e. friends, individuals with food allergies, internet sources, medical staff), participants revealed only trusting certain sources, imparted by medically informed individuals, or that provided by Food Allergy Canada:

I think in terms of the trusted source, you have to have information that is credible, accurate, based on facts and based on scientific research that's been medically vetted. (Participant 8)

While all participants required relevant information to inform their organizational approaches to care and develop informed guidelines, all discussed concerns surrounding the lack of coherent and consistent food allergy information:

I have my stacks of scientific research papers and then just when I feel like I've come up with a number [food allergy prevalence] that makes sense, something else gets published or I come across some different source. (Participant 6)

It depends on the allergy or information that you need. There's no common source for information on all allergies. You need to have knowledge of different sources, or keep searching for proper information. (Participant 3)

Acquiring realistic and accurate information was of paramount concern for participants and jeopardized their institutional credibility. These concerns were felt by many, expressing inconsistencies in data reporting, impacting their organization's integrity and further affecting the population they serve:

So if someone's just Googling, they can get an old report and then old statistics... then you get questioned by a group of citizens and it becomes very destabilizing. (Participant 2)

I'm trying to be authoritative and the information that you're getting is... just... not, it's almost like a moving target. (Participant 5)

\section{Descriptions and Experiences of Food Allergy in Canada: Where did it come from? Is it increasing?}

While reflecting on the current landscape of food allergies in Canada, many participants remarked on their pervasiveness, reflecting the normalization of the food allergy experience for so many Canadians:

It just seems like in every aspect of our environment somebody is affected or influenced. (Participant 1)

Participants expressed their perception was rooted in the 
increased attention and awareness directed towards food allergies in numerous settings (i.e. restaurants, schools). While inherently incapable of forming definite understandings of food allergies due to inconsistent information, many participants revealed:

I think your... perception is also influenced by the fact, you know, if you have one child in the school with any allergy, the entire school becomes very aware. So now you have the entire school board and jurisdiction who will take it on to protect a few. And every restaurant you go to has an insert for the people with food allergies. So everywhere you go, it's being reinforced on you every time. (Participant 7)

... and so the next, very logical assumption, "Hey, 10 years ago no one had food allergy. Tonight I'm working [at a restaurant] and have to deal with 15 people who had allergies, rightfully, it's on the rise. (Participant 8)

Others expressed the view that the perceived increase in food allergies was actually an artifact. This was grounded in participants' understanding that perhaps food allergies are not actually increasing but have been granted more attention in the past decade:

"Maybe it's not increasing, maybe we're just talking about it more". (Participant 6)

\section{Published Findings}

When queried about the results of the national Canadian survey, participants reported being very excited about and pleased with the work, reporting that the information provided more credibility on the issue of food allergy in Canada and would be able to facilitate improved care for those living with food allergies:

I was happy to see this. I thought it finally gave some voice and some urgency to allergies 'cause for so long it's been, "Oh, it's just your allergies", you know, now people are taking it more seriously and understanding that it's very impactful. (Participant 6)

Participants also indicated this new information would significantly aid their organizations. Many highlighted that the findings would assist in building institutional credibility while aiding the development of food allergy policy and practice:

I think we're closer to getting at the realistic burden of illness. Understanding that information is important because it would link to priorities we have at the moment... My office has been trying to utilize different aspects to translate and find ways of better communicating with groups. (Participant 2)

Many participants noted how this recent information conferred a greater sense of credence and integrity for their organization as they could now quote recent, valid prevalence numbers which contributed to their ability to facilitate awareness and advocacy:
If we have statistics that back up the information, it helps to build the story. There's a need out there for information, so the more there is, the more we can effectively educate and raise food allergy awareness. But advocacy is huge 'cause anytime we're trying to get government attention or industry attention you've got to give them the scope of the problems. So this is helpful. (Participant 1)

Along with facilitating institutional capacity, participants underscored the pertinence of the results for large government health organizations, suggesting the study results would aid them in their understanding of precise food allergy numbers, while ensuring applicable guidelines were developed and tailored accordingly:

Some have the responsibility to set appropriate guidelines, understand them and to know acceptable risk in the country, so with this information groups can better protect as many people as possible. Others work with groups in all government jurisdictions to develop appropriate health policies, but public health agencies on the ground need the information the most. They always go to those who develop the guidelines to put things in context so it would be important for government institutions to know accurate information to put things in context for public health units. (Participant 3)

\section{Participant Recommendations for Future Research}

While participants were pleased with the initial results from the study, there was the desire for more research related to a range of topics (i.e. increases in prevalence, impact on quality of life, the cultural discourse surrounding food allergies) to facilitate a comprehensive understanding of food allergies in Canada. This sentiment was expressed by all participants. With more data related to food allergy, participants would be able to provide increased and more relevant information to their constituents, develop targeted approaches, and facilitate credible guidelines and policies:

And when you have actual numbers, it makes you much more credible, especially when they're hearing the same numbers from everyone. (Participant 4)

This feeling was reiterated by all participants, suggesting that relevant consistent information would enhance their ability to promote food allergy guidelines and enhance evidence-based policy development. Given that many participants assist health institutions in the development of guidelines and the implementation of patient care programs, many expressed the concern for improved information:

I was at the Canadian Food Inspection Agency food labelling meeting in Toronto and one of the themes that came out from that was a huge consensus around the need for regulation around food allergies; more information that would help with policy. (Participant 2) 
Participants realized the impact food allergies had on the general public and their ability to enjoy life. Participants often discussed the negative impact of food allergies on an individual's quality of life indicating this concern should be captured in future work to facilitate a greater discussion on the social and psychological impacts of food allergy:

Could we look from a quality of life perspective 'cause, if that's a focus in terms of when we talk about choices, for example, when you send your kids to school or daycare that have an allergy policy that's aware of these concerns. Or when you're travelling or dining out, 'cause food allergic consumers always think of these things. We're hearing that their whole quality of life is impacted. (Participant 5)

Recognizing the impact of food allergy on one's quality of life, participants suggested the need to target aspects related to the societal discourse surrounding food allergies. This would unearth information on public sensitivity and responsiveness to those with food allergies. By facilitating an understanding of cultural discourse in the Canadian context, participants suggested they would be able to have a greater understanding of societal values and tailor programs to suit the needs of those affected by food allergy:

...One of the thoughts I had was around the piece of understanding the cultural discourse at one present time versus another and how that... discourse may or may not change "cause it will speak to the values that go along with it, right. And if you ask them something like, "How do you... what do they think caused their allergy?" (Participant 4)

\section{Discussion \& Conclusion}

These results demonstrate the perspectives of food allergy knowledge users in Canada, highlighting the importance of $\mathrm{iKT}$ in the context of knowledge creation. Knowledge users reported a lack of access to (accurate) knowledge produced by researchers (i.e. data inconsistencies, accessibility challenges). This aligns with the literature, which suggests the knowledge to action gap occurs due to problems with the knowledge created as well as the failure to engage knowledge users [13]. Indeed, confusion and heightened uncertainty around information resources pervaded the discussion, underlying the issue that knowledge is ineffectively translated in accessible ways leading to deficiencies in the ability of knowledge users to provide resources for their constituents. This is turn hindered organizational credibility and limited the ability of knowledge users to move research to practice. Similar conclusions have been drawn in other contexts, calling for researchers to better understand the informational needs of knowledge users $[1,14,30,31]$. With respect to research directions, these knowledge users pointed to the need for more information regarding the (potential) rise in food allergy prevalence, impacts on families and quality of life, and the need to create empathy in the non-allergic community in order to maximize choice and minimize risk for those affected by food allergy in Canada. While our third national survey cannot answer all these questions, we can certainly address issues of risk perception and prevalence, leaving the questions of impact for follow up qualitative investigations [indeed, much has already been done in this area; see 19, 23, 24].

While iKT approaches have tended to be practiced by "researchers in some fields for some problems" [13], providing knowledge users with the opportunity to participate in knowledge creation is an essential step for redirecting questions, acquiring previously unsought information, and tailoring approaches to information acquisition [8]. As these results indicate, knowledge users felt strongly about being integrated into the research process, as they had the ability to query findings, pose and redirect inquiries, and offer suggestions to best suit their organizational needs. Not only does this help facilitate stronger research outcomes, it also provides the opportunity to develop stronger relations between knowledge users and researchers, and ultimately inform evidence based practice. As others have stressed, iKT provides research benefits that are more practice and policy relevant while also increasing mutual understandings of roles and values among decision makers [32]. The need to develop integrated plans around knowledge user involvement at each step of the research process, as opposed to concentrating on moving research findings into practice at "end of grant" $[7,30]$ is required in order to promote more efficient health care provision and meet the needs of both knowledge users and the broader community; that is, to make science useful.

\section{REFERENCES}

[1] Innvaer S, Vist G, Trommald M, Oxman A. Health policy-makers' perceptions of their use of evidence: a systematic review. J Health Serv Res Policy 2002; 7:239-244.

[2] Ouimet M, Landry R, Amara N. Belkhodja O. What factors induce health care decision-makers to use clinical guidelines? Evidence from provincial health ministries, regional health authorities and hospitals in Canada. Soc Sci Med 2006; 62:964-976.

[3] Lomas J. Using 'linkage and exchange' to move research into policy at a Canadian foundation. Health Aff 2000; 19:236-240.

[4] Graham ID, Logan J, Harrison MB, Straus SE, Tetroe J, Caswell W, \& Robinson, N. Lost in knowledge translation: time for a map? Journal of continuing education in the health professions 2006; 26(1), 13-24.

[5] Government of Canada. (2014, February, 27). Canadian Institutes of Health Research. More About Knowledge Translation at CIHR. Retrieved from http://www.cihr-irsc.gc. ca/e/39033.html\#Definition 
[6] Kothari A, Wathen CN. A critical second look at integrated knowledge translation. Health Policy, 2013; 109(2):187-191.

[7] Gagnon ML. Moving knowledge to action through dissemination and exchange. J Clin Epidemiol2011; 64:25-31.

[8] Field B, Booth, A, Ilott, I. \& Gerrish K. Using the Knowledge to Action Framework in practice: a citation analysis and systematic review. Implement Sci, 2014; 9(1), 172-186.

[9] Oborn E, Barrett, M, Prince K, Racko G. Balancing exploration and exploitation in transferring research into practice: a comparison of five knowledge translation entity archetypes. Implement Sci 2013; 8:104.

[10] Wilkinson H, Gallagher M, Smith M. A collaborative approach to defining the usefulness of impact: lessons from a knowledge exchange project involving academics and social work practitioners. Evid Policy 2012; 8(3):311-327.

[11] Allender S, Nichols M, Foulkes C, Reynolds R, Waters E, King L, Gill T, Armstrong R, Swinburn B. The development of a network for community- based obesity prevention: the CO-OPS Collaboration. BMC Public Health 2011; 11:132.

[12] Tee S, Bockle E. Closing the gap - A partnership approach to community care education for long term conditions. Nurse Edu Today 2012; 32:822-828.

[13] Bowen SJ, Graham ID. From knowledge translation to engaged scholarship: Promoting research relevance and utilization. Arch Phys Med Rehabil 2013; 94(1 Suppl): S3-S8.

[14] Dobbins M. Jack, S. Thomas H. \& Kothari A. Public Health Decision - Makers' Informational Needs and Preferences for Receiving Research Evidence. Worldviews on Evidence Based Nursing, 2007; 4(3), 156-163.

[15] Tetroe JM, Graham, ID, Foy R, Robinson N, Eccles MP, Wensing M. \& Grimshaw JM. Health research funding agencies' support and promotion of knowledge translation: an international study. Milbank Quarterly 2008; 86(1), 125-155.

[16] Palinkas LA and Soydan H. Translation and Implementation of Evidence-Based Practice. Oxford: Oxford University Press, 2012

[17] Soller L, Ben-Shoshan M, Harrington, DW, Knoll M, Fragapane J, Joseph L, St. Pierre Y, La Vieille S, Wilson K, Elliott SJ, Clarke AE. Prevalence and predictors of food allergy in Canada: a focus on vulnerable populations. The Journal of Allergy and Clinical Immunology: In Practice 2015; 3(1), 42-49.

[18] Soller L., Ben-Shoshan, M., Harrington, D. W., Fragapane J, Joseph L, St Pierre Y. \& Clarke, AE. Overall prevalence of self-reported food allergy in Canada. Journal of Allergy and Clinical Immunology 2012; 130(4), 986-988.

[19] Harrington DW, Elliott SJ, Clarke AE, Ben-Shoshan, M., \& Godefroy, S. Exploring the determinants of the perceived risk of food allergies in Canada. Human and Ecological Risk Assessment: An International Journal 2012; 18(6),
$1338-1358$.

[20] Sampson HA, Gerth van Wijk R, Bindslev-Jensen C, Sicherer $\mathrm{S}$, Teuber SS, Burks A. \& Werfel T. Standardizing double-blind, placebo-controlled oral food challenges: American Academy of Allergy, Asthma \& ImmunologyEuropean Academy of Allergy and Clinical Immunology PRACTALL consensus report. Journal of Allergy and Clinical Immunology 2012; 130(6), 1260-1274.

[21] Rahaman T, Vasiljevic T, \& Ramchandran L. Conformational changes of $\beta$-lactoglobulin induced by shear, heat, and $\mathrm{pH}-$ Effects on antigenicity. Journal of dairy science 2015; 98(7), 4255-4265.

[22] Dean, J., Fenton, N. E., Shannon, S., Elliott, S. J., \& Clarke, A. (2015). Disclosing food allergy status in schools: health related stigma among school children in Ontario. Health \& social care in the community 2015. doi: 10.1111/hsc.12244

[23] Fenton NE, Elliott SJ, Cicutto L, Clarke AE, Harada L, \& McPhee E. Illustrating Risk: Anaphylaxis Through the Eyes of the Food - Allergic Child. Risk Analysis 2011; 31(1), 171-183.

[24] Minaker LM, Elliott SJ, \& Clarke A. Low income, high risk: the overlapping stigmas of food allergy and poverty. Critical Public Health 2014; (ahead-of-print), 1-16.

[25] Sicherer SH, Munoz-Furlong A, Godbold JH, Sampson HA. US prevalence of self-reported peanut, tree nut, and sesame allergy: 11-year follow-up. Journal of Allergy and Clinical Immunology 2010; 125:1322-6.

[26] Venter C, Hasan AS, Grundy J, et al. (2010). Time trends in the prevalence of peanut allergy: three cohorts of children from the same geographical location in the UK. Allergy Jan; $65: 103-8$

[27] Green J, \& Thorogood N. Qualitative methods for health research 2013; Sage.

[28] Crabtree BF, \& Miller WL. (Eds.). Doing qualitative research 1999; Sage Publications.

[29] Strauss, A. L., \& Corbin, J. M. (1998). Basics of Qualitative Research: Grounded theory procedures and techniques (2nd ed.). Newbury Park, CA: SAGE

[30] Baxter J, \& Eyles J. Evaluating qualitative research in social geography: Establishing 'rigour' in interview analysis. Transactions of the Institute of British Geographers 1997; 22(4), pp. 505-525.

[31] Lavis JN. Assessing provincial or national efforts to link research to action. In Policy Decision-Making 2006; Ontario, Canada: McMaster University.

[32] Eriksson, C. C., Fredriksson, I., Fröding, K., Geidne, S., \& Pettersson, C. (2014). Academic practice-policy partnerships for health promotion research: Experiences from three research programs. Scandinavian journal of public health, 42(15 suppl), 88-95. 